\title{
Rescue of a case of ventricular septal defect with Eisenmenger syndrome using an extracorporeal membrane oxygenator
}

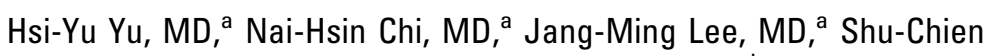 \\ Huang, MD, ${ }^{a}$ Wen-Je Ko, MD, ${ }^{a}$ Jou-Kou Wang, MD, ${ }^{b}$ Yung-Chie Lee, \\ MD, ${ }^{a}$ Shoei-Shen Wang, MD, ${ }^{a}$ Fang-Yue Lin, MD, a and Yih-Sharng \\ Chen, MD, ${ }^{a}$ Taipei, Taiwan
}

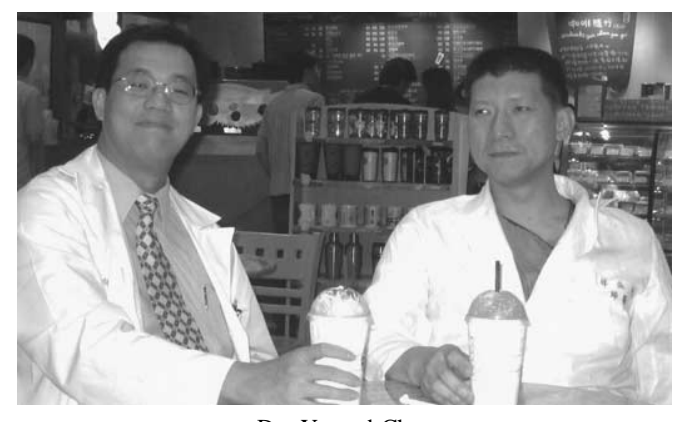

 isenmenger syndrome with ventricular septal defect (VSD) complicated with acute deterioration is always fatal because no treatment modality is reported effective. The present article reports our experience to rescue such a case by modern therapeutic modalities.

\section{Clinical Summary}

A 38-year-old male patient presented with severe respiratory distress. A large VSD with Eisenmenger syndrome had been diagnosed in the patient 2 years previously (Figure 1). A chest x-ray film revealed a pulmonary hemorrhage at the upper lobe of the right lung (Figure 2, $A$ ). The patient's arterial oxygen concentration was extremely low (68\%) even with ventilator support of $100 \%$ oxygen. A venovenous extracorporeal membrane oxygenator (ECMO) was inserted through the left femoral vein (outflow from the patient) and right jugular vein (inflow to the patient) in an attempt to increase his oxygen saturation. Because of severe mixing of blood across the VSD, ${ }^{1}$ the percutaneous saturation was still low $\left(\mathrm{SpO}_{2}=74 \%\right)$ even with the venovenous ECMO support. Intermittent bradycardia occurred during the support, which was considered to be the result of inadequate tissue oxygenation. It seemed critical to find a way to decrease the mixing of deoxygenated blood across the VSD to save the patient's life. Two days later, after a discussion with the patient's family, we decided to perform an operation to close the VSD and create an atrial septal defect (ASD) with the use of cardiopulmonary bypass and aortic crossclamping. To prevent right-sided heart failure after the open operation, myocardial temperature was kept at $37^{\circ} \mathrm{C}$ with warm antegrade and retrograde cardioplegic solution. ${ }^{2}$ The large perimembranous VSD without aortic overriding was closed with a knitted Dacron patch and continuous suture with 4-0 polypropylene. A $1.5 \times$ $1.5 \mathrm{~cm}^{2}$ fenestration at the atrial level was created to prevent congestion of the right side of the heart after closure of this large VSD. The operation was completed smoothly without right-sided heart failure developing. The patient was sent back to the intensive care unit with

From the Departments of Surgery ${ }^{\mathrm{a}}$ and Pediatrics, ${ }^{\mathrm{b}}$ National Taiwan University Hospital and National Taiwan University College of Medicine, Taipei, Taiwan.

Received for publication Aug 29, 2003; revisions requested Oct 3, 2003; accepted for publication Oct 10, 2003.

Address for reprints: Yih-Sharng Chen, MD, Department of Surgery, National Taiwan University Hospital, No 7, Chung-Shan S. Road, Taipei,

Taiwan (E-mail: yschen11@yahoo.com.tw).

J Thorac Cardiovasc Surg 2004;127:582-3

$0022-5223 / \$ 30.00$

Copyright $\odot 2004$ by The American Association for Thoracic Surgery doi:10.1016/j.jtcvs.2003.10.027

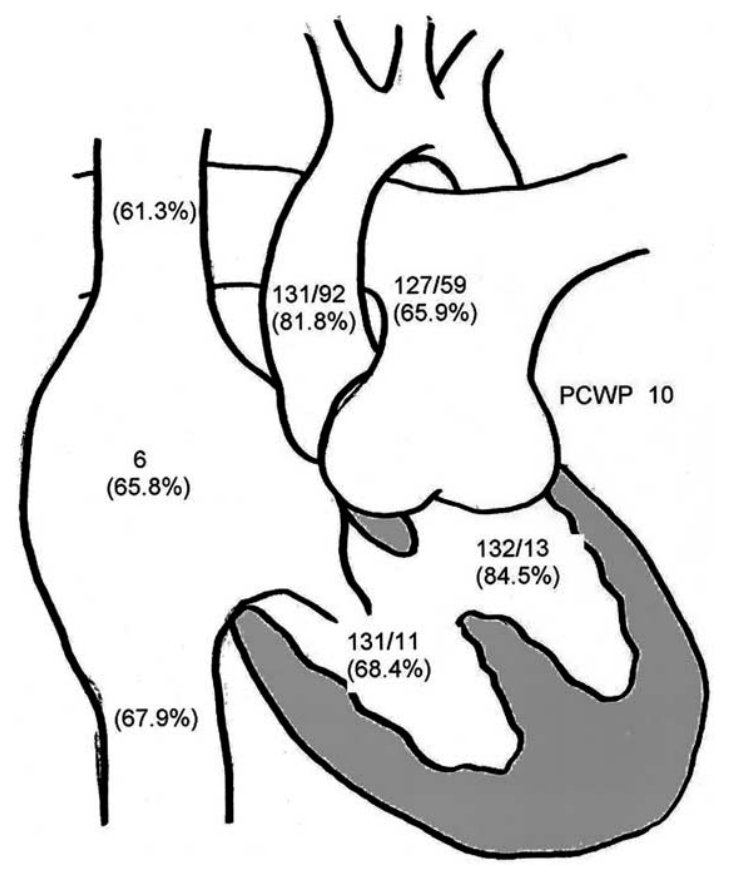

Figure 1. Data of cardiac catheterization 2 years before this episode. Listed data represent pressure and oxygen saturation, respectively. Severe pulmonary hypertension equal to the aortic pressure with low systemic oxygen saturation was found. Flow ratio between aorta and pulmonary artery was approximately equal to 1.2 with the assumption that oxygen saturation in the left atrium was the same as that in the left ventricle (84.5\%). Preserved right-sided heart function was suggested by the low right atrial pressure and mild degree of tricuspid regurgitation. PCWP, Pulmonary capillary wedge pressure.

the venovenous ECMO and systemic oxygen saturation increased to more than $90 \%$.

During the waiting period for lung transplantation, he was maintained with the venovenous ECMO and nitric oxide inhalation. His clinical condition improved gradually. Another episode of pulmonary hemorrhage occurred 20 days after the use of the ECMO (Figure 2, B). The dose of heparin was decreased to keep the activated clotting time (ACT) between 120 and 140 seconds instead of the usual range (160-180 seconds). This episode resolved 5 days later. Two days later, a blood clot in the right lung was evacuated by a thoracoscopic procedure to create lung expansion and decrease the pulmonary vascular resistance.

Forty-six days after venovenous ECMO use, a suitable donor was found and cadaveric unilateral left lung transplantation was performed 

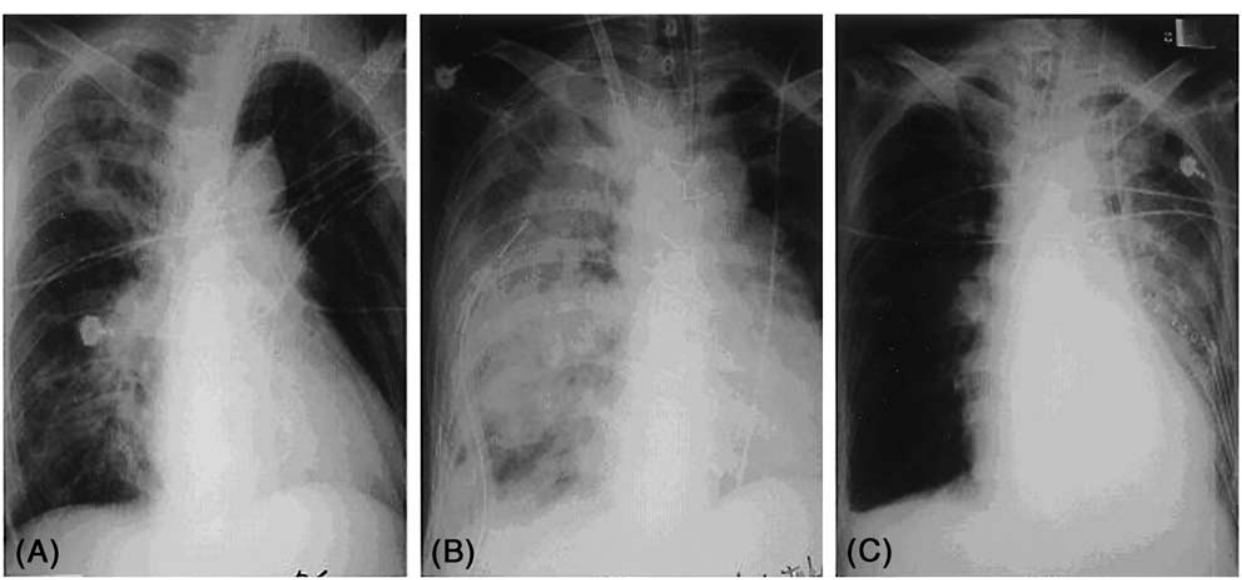

Figure 2. A, First episode of pulmonary hemorrhage in the right upper lung with severe oxygen desaturation. Pulmonary hypertension was evidenced by the dilated right pulmonary trunk. B, Another episode of pulmonary hemorrhage $\mathbf{2 0}$ days after venovenous ECMO use. Inflow cannula was seen in the superior vena cava. C, Five days after transplantation of left lung when ASD was closed and ECMO was removed.

smoothly with the use of a venoarterial $\mathrm{ECMO}^{3}$ (we shifted the ECMO from venovenous to venoarterial in the operating room). Left lung transplantation was performed because multiple nodules were found on the donor's right lung. Three days later, the ASD was closed with an occluder (Amplatzer septal occluder; AGA Medical Corporation, Minneapolis, Minn) to decrease right-sided heart overflow and donor lung edema by the left-to-right shunt through ASD (Qp/Qs before ASD occlusion was estimated to be 1.4). Two days later, the patient was weaned from the venoarterial ECMO with a systemic oxygen concentration of $97 \%$ and pulmonary arterial pressure of $50 / 20 \mathrm{~mm} \mathrm{Hg}$ (Figure 2, C). The patient was weaned from the respirator 2 weeks later and discharged 3 weeks after that.

\section{Discussion}

To our knowledge, this is the first report of a case of Eisenmenger syndrome with hypoxic attack successfully rescued by the use of an ECMO and lung transplantation. One of the most frequent complications of Eisenmenger syndrome is hypoxic attack caused by pulmonary hemorrhage, ${ }^{4,5}$ which is regarded as a fatal condition because of the complex clinical situation. The success of the presenting case can be attributed to the systemic planning in the beginning and the help of modern medical equipment.

We chose a venovenous ECMO because, from our experience, a venoarterial ECMO can only serve as a temporary rescue tool and cannot be maintained more than 2 weeks. The main adverse effect beyond that period is the gradual development of multiorgan failure caused by malperfusion of end organs, which is supposed to be related to the loss of aortic pulsatility. A venovenous ECMO is a safer tool to provide air exchange without marked influence on aortic pulsatility and thus can be used for a prolonged period.

Another controversial decision was the timing of closing the VSD in this case. We decided to close the VSD in that early stage because, according to the report of Stojnic and colleagues, ${ }^{1}$ the shunt flow pattern across the VSD in patients with Eisenmenger syndrome is a combination of a left-to-right shunt in the early systolic period and a right-to-left shunt in the late systolic to diastolic period, with an overall Qp/Qs equal to 1 $(1.1 \pm 0.4$ in that study) and a blood mixing percentage of approximately $24 \%$. Therefore the closure of VSD, in theory, decreases the mixing of deoxygenated and oxygenated blood but does not increase the workload of the right ventricle. We also created an atrial fenestration to prevent the potential development of right-sided heart failure associated with the operative procedure. The clinical situation was exactly as we expected: The patient's oxygen saturation increased from $68 \%$ to more than $90 \%$ whereas the central venous pressure level was equal before and after the operation. This patient was fortunate to have preserved right-sided heart function under such high pulmonary resistance, reflected by the low central venous pressure level and mild degree of tricuspid regurgitation.

\section{Conclusion}

As shown by the presenting case, VSD with Eisenmenger syndrome complicated with pulmonary hemorrhage and hypoxic attack is a potentially rescuable condition with the use of modern medical equipment. Lung or heart-lung transplantation is still the final answer for this condition.

\section{References}

1. Stojnic B, Pavlovic P, Ponomarev D, Aleksandrov R, Prcovic M. Bidirectional shunt flow across a ventricular septal defect: pulsed Doppler echocardiographic analysis. Pediatr Cardiol. 1995;16:6-11.

2. Kulshrestha P, Rousou JA, Engelman RM, Flack JE 3rd, Deaton DW, Wait RB, et al. Does warm blood retrograde cardioplegia preserve right ventricular function? Ann Thorac Surg. 2001;72:1572-5.

3. Ko WJ, Chen YS, Lee YC. Replacing cardiopulmonary bypass with extracorporeal membrane oxygenation in lung transplantation operations. Artif Organs. 2001;25:607-12.

4. Young D, Mark H. Fate of the patient with the Eisenmenger syndrome. Am J Cardiol. 1971;28:658-69.

5. Niwa K, Perloff JK, Kaplan S, Child JS, Miner PD. Eisenmenger syndrome in adults: ventricular septal defect, truncus arteriosus, univentricular heart. J Am Coll Cardiol. 1999;34:223-32. 